\title{
PREVALÊNCIA DE LESÃO ENDODÔNTICA EM PACIENTES DIABÉTICOS
}

\author{
Prevalence of apical periodontitis in diabetic patients \\ Prevalencia de lesión endodóntica de pacientes diabeticos
}

\section{RESUMO}

Objetivo: Investigar a prevalência de lesões endodônticas em pacientes diabéticos e não diabéticos. Métodos: Em estudo transversal, exames radiográficos (panorâmica e seriografia) de 80 pacientes, sendo 40 diabéticos tipo II e 40 não diabéticos, receberam avaliação, por meio de um índice de escores periapicais, das regiões perioendodônticas de todos os elementos dentais presentes. Os dados foram analisados pelo programa BioEstat 5.3 ${ }^{\circledR}$. Resultados: Encontrou-se pelo menos 1 dente apresentando lesão apical em $90 \%$ $(n=32)$ dos pacientes diabéticos e $52 \%(n=21)$ dos pacientes não diabéticos $(p=0,0001)$. Quanto aos dentes tratados endodonticamente, nos pacientes diabéticos, foram encontrados $44 \%(n=51)$ com lesões endodônticas, e apenas $17 \%(n=17)(p=0,0004)$ no grupo controle. Os pacientes diabéticos apresentaram maior quantidade de lesões endodônticas em relação aos pacientes não diabéticos ( $\mathrm{p}=0,0189$ ). Conclusão: De acordo com esses resultados, podese concluir que a Diabetes Mellitus tipo II está associada ao aumento da prevalência de lesões endodônticas.

Descritores: Doenças Periapicais; Diabetes Mellitus; Endodontia.

\begin{abstract}
Objective: To investigate the prevalence of apical periodontitis $(A P)$ in diabetic and nondiabetic patients. Methods: Cross-sectional study, in which the radiographic records (panoramic and full-mouth periapical radiographs) of 80 patients, being 40 type II diabetic patients and 40 nondiabetic subjects, had the periapical and endodontic regions of all teeth present evaluated by means of the periapical index score. Data was analyzed through BioEstat $5.3^{T M}$ software. Results: At least one tooth was found with apical periodontitis in $90 \%(n=32)$ of the diabetic patients and in 52\% $(n=21)$ of nondiabetic subjects $(p=0.0001)$. Regarding root-filled teeth, 44\% ( $n=51)$ presented AP amongst the diabetic patients, whereas only $17 \%(n=17)(p=0.0004)$ were affected in the control group. The diabetic patients presented larger quantity of apical periodontitis than did the nondiabetics $(p=0.0189)$. Conclusion: According to these results, type 2 Diabetes Mellitus is associated with an increase in the prevalence of apical periodontitis.
\end{abstract}

Descriptors: Apical periodontitis; Diabetes Mellitus; Endodontics.

\section{RESUMEN}

Objetivo: Investigar la prevalencia de lesiones endodónticas de pacientes diabéticos e no diabéticos. Métodos: Estudio transversal con pruebas radiológicas (panorámica y seriografia) de 80 pacientes, siendo 40 diabéticos tipo II y 40 no diabéticos que recibieron evaluación a través del índice de puntuaciones periapicales de las regiones perioendodónticas de todos los elementos dentales presentes. Los datos fueron analizados con el programa BioEstat 5.3. Resultados: Se encontró por lo menos un diente con lesión apical en el 90\% $(n=32)$ de los pacientes diabéticos y el 52\% $(n=21)$ de los no diabéticos $(p=0,0001)$. Respecto a los dientes tratados endodónticamente, el 44\% $(n=51)$ de los pacientes diabéticos presentó lesiones endodónticas y solamente el 17\% $(n=17)(p=0,0004)$ en el grupo control. Los pacientes diabéticos presentaron más lesiones endodónticas que los no diabéticos ( $p=0,0189)$. Conclusión: A partir de los resultados se concluye que la Diabetes Mellitus tipo II está asociada con el aumento de la prevalencia de lesiones endodónticas.

Descriptores: Enfermedades Periapicales; Diabetes Mellitus; Endodoncia.
Artigo Original
1) Universidade de Fortaleza - UNIFOR Fortaleza (CE) - Brasil

2) Cirurgião-dentista pela Universidade de Fortaleza - UNIFOR Fortaleza (CE) - Brasil 


\section{INTRODUÇÃO}

A Diabetes Mellitus é uma doença sistêmica crônica que engloba sistemas metabólicos, vasculares e endócrinos, provavelmente de caráter hereditário, consequente à deficiência parcial ou total de insulina, o que acarreta uma inadequada utilização dos carboidratos e alterações no metabolismo lipídico e proteico ${ }^{(1-5)}$.

Essa alteração sistêmica resulta em uma profunda ou absoluta deficiência de produção de insulina - tipo I, dependentes de insulinoterapia diária (correspondendo a $5-10 \%$ dos casos), ou resistência do tecido aos seus efeitos metabólicos celulares - tipo II, não insulino dependente, o que ocorre em $90 \%$ dos casos, nos quais o paciente faz o controle de sua glicemia apenas com dietas, sendo a insulina utilizada em casos excepcionais ${ }^{(5-7)}$.

A Diabetes Mellitus afeta algumas funções do sistema imune e está associada com falhas do processo de cicatrização tecidual e comprometimento de respostas imunes $^{(7,8)}$. Estudos estatísticos demonstram que a diabetes está diretamente relacionada às elevadas taxas de mortalidade nos Estados Unidos ${ }^{(9,10)}$. No Brasil, estima-se que essa doença já atinja 13 milhões de indivíduos ${ }^{(2,11)}$.

As complicações orais em pacientes com diabetes não controlada podem incluir xerostomia, cicatrização deficiente, infecções, aumento da incidência e severidade de cáries, candidíase, gengivite e periodontite ${ }^{(12)}$. Doenças periodontais severas têm sido associadas a elevados níveis séricos de glicose ${ }^{(13)}$, especialmente em pacientes não compensados $^{(12)}$. Demonstra-se que pacientes diabéticos apresentam elevadas perdas de inserção periodontal $l^{(9)}$, ocorrendo inclusive contraindicações em casos nos quais são necessários implantes dentários para promover reabilitação protética ${ }^{(14)}$.

As perdas ósseas apicais, chamadas de lesões endodônticas ou periodontites apicais, visualizadas radiograficamente como áreas radiolúcidas, constituem a primeira sequela relativa à contaminação do sistema de canais radiculares como consequência da cárie dental não tratada ${ }^{(15)}$ ou de dentes tratados endodonticamente e não restaurados adequadamente ${ }^{(16)}$. Investigações epidemiológicas têm demonstrado elevadas prevalências de lesões endodônticas, variando de $1,4 \%^{(17)}$ a $8,0 \%{ }^{(18)}$, utilizando dentes como unidades de estudo. Quando indivíduos são utilizados como unidades, a prevalência mostra-se acima de $61,1 \%$, aumentando com a idade ${ }^{(15,19)}$.

O tratamento endodôntico é o de eleição para os casos em que ocorrem lesões endodônticas, mas esses tratamentos devem ser acompanhados clínica e radiograficamente, para que se possa avaliar de forma segura o sucesso ou o insucesso do caso ${ }^{(20,21)}$. Entretanto, a literatura que associa à patogenia progressão e reparo de patologias endodônticas mostra-se marcadamente vaga ${ }^{(17,18)}$.

Além disso, revelam-se estudos que avaliaram clinicamente a possibilidade de associação entre Diabetes Mellitus e lesões endodônticas ${ }^{(22-24)}$. A temática desperta interesse por ser pouco investigada. Dessa forma, o objetivo do presente estudo foi investigar a prevalência de lesões endodônticas em pacientes diabéticos e não diabéticos.

\section{MÉTODOS}

Trata-se de estudo quantitativo, transversal e retrospectivo, no qual foram utilizados dados de prontuários e imagens radiográficas do banco de pacientes do curso de Odontologia e do Núcleo de Atenção Médica Integrada (NAMI) da Universidade de Fortaleza (UNIFOR). Estudaram-se os prontuários e as radiografias (panorâmicas e periapicais), do período compreendido entre 2000-2011, de 80 pacientes, sendo 40 portadores de Diabetes Mellitus tipo II (13 homens e 27 mulheres, com idades variando entre 37 e 68 anos) e 40 não diabéticos (16 homens e 24 mulheres, com idades variando entre 35 e 70 anos), cuja coleta ocorreu em 2011.

Com o objetivo de diminuir a influência da doença periodontal no presente estudo, pacientes que apresentavam abaixo de 7 dentes na arcada dentária foram excluídos, por encontrarem-se classificados como doença periodontal severa $^{(15)}$. Todos os pacientes diabéticos tipo II que não apresentaram o critério de exclusão periodontal tiveram suas radiografias analisadas. Com relação aos indivíduos não diabéticos, a escolha foi aleatória junto ao banco de dados citado anteriormente.

De posse dos 80 prontuários dos pacientes, três examinadores calibrados preencheram, inicialmente, uma planilha Excel contendo dados individuais dos pacientes: (i) número de dentes presentes, (ii) número de lesões endodônticas, (iii) número de dentes tratados endodonticamente, (iv) número de dentes tratados endodonticamente com lesões endodônticas, (v) número de dentes sem tratamento endodôntico e com lesões endodônticas. Levou-se em consideração o tempo de tratamento e a história dental pregressa, pois, através desta, saber-se-ia se o dente em análise apresentava lesão apical antes do tratamento endodôntico. Através dos prontuários, pôde-se fazer uma correlação do tempo de tratamento com a imagem radiográfica presente. Nos casos em que não havia - ou as imagens radiográficas não apresentavam - condições adequadas de avaliação (imagem comprometida) ou as avaliações eram datadas com mais de 2 anos, os pacientes eram convidados a realizar novas radiografias (panorâmica e periapicais). 
Em seguida, procedeu-se à análise das imagens radiográficas, e, inicialmente, todos os dentes foram categorizados como tratados endodonticamente ou não, para que se pudesse conceituá-los de acordo com os escores do Índice de Escores Periapicais, da escala PAI ${ }^{(25)}$ (index periapical), apresentado na Tabela 1 . Todos os dentes que apresentavam a cavidade pulpar preenchida com material radiopaco receberam a classificação de "tratado endodonticamente".

Realizou-se, então, a avaliação das radiografias por cada examinador, sendo repetida após 7 e 14 dias, em diferentes sequências, para testar a validade dos dados. Por meio de um negatoscópio apropriado, com aumento de 3,5x, realizouse a observação das radiografias, para que se facilitasse a visualização dos detalhes das estruturas periapicais. Cada escore usado na escala PAI representou um dado numérico relativo ao estágio de evolução da lesão apical ${ }^{(25)}$ - valores obtidos na escala PAI $>2$ sinalizam presença de patologia apical. Os dados considerados com diferentes escores pelos examinadores receberam nova análise em conjunto para nova conceituação na escala PAI.

Os resultados obtidos foram tabulados e analisados estatisticamente através do programa BioEstat $5.3^{\circledR}$ para Windows (Instituto Marimauá, AM, Brasil), utilizando-se o teste Wilcoxon quando os indivíduos foram as unidades de análise, e o teste Qui-Quadrado quando as unidades de análise eram os dentes.

$\mathrm{O}$ projeto de pesquisa do presente estudo recebeu aprovação do Comitê de Ética em Pesquisa com Seres Humanos (COETICA) da Universidade de Fortaleza (UNIFOR), sob o parecer $n^{\circ}$ 172/2008.

\section{RESULTADOS}

Encontraram-se as médias dos números de dentes

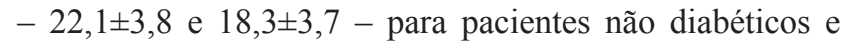
diabéticos, respectivamente $(\mathrm{p}=0,0001)$. A presença de lesões endodônticas em pelo menos um dente ocorreu em $90 \%(\mathrm{n}=32)$ dos pacientes diabéticos e em 52,5\% ( $\mathrm{n}=21)$ dos pacientes não diabéticos $(\mathrm{p}=0,0001)$.

Um ou mais dentes tratados endodonticamente foram igualmente encontrados em ambos os grupos (97,5\%), em 39 pacientes em cada grupo $(p>0,05)$. Entre os pacientes diabéticos, 77,5\% $(\mathrm{n}=31)$ dos dentes tratados endodonticamente apresentavam lesões endodônticas, enquanto no grupo controle esse fato ocorreu em $40 \%$ $(\mathrm{n}=16)$ dos casos $(\mathrm{p}=0,03)$ (Tabela II).

Com relação à análise do total de dentes, avaliaram-se 1.620 dentes, sendo 734 de pacientes diabéticos, dos quais $12,1 \%(\mathrm{n}=89)$ apresentavam lesões endodônticas $(\mathrm{PAI} \geq 3)$, com alterações na estrutura óssea e formação de lesões visíveis radiograficamente. No grupo controle, esse tipo de alteração também foi a mais frequente (PAI $\geq 3)$, uma vez que, dos 886 dentes avaliados, 4,1\% (n=37) apresentaram lesões endodônticas. Os pacientes diabéticos apresentaram maior quantidade de lesões endodônticas em relação aos não diabéticos $(\mathrm{p}=0,0189)$.

Tabela I - Índice de Escores Periapicais - Escala PAI*

\begin{tabular}{ll}
\hline Escore & Critérios \\
\hline 1 & Estruturas periapicais normais \\
2 & Pequena alteração na estrutura óssea \\
3 & Alterações na estrutura óssea com formação de \\
& lesão \\
4 & Lesão com área radiolúcida bem definida \\
5 & Lesão extensa com borda difusa \\
\hline
\end{tabular}

*Adaptado para o português. Ørstavik D, Kerekes K, Eriksen HM. The periapical index: a scoring system for radiographic assessment of apical periodontitis. Endod Dent Traumatol. 1986;2(1):20-34.

Tabela II - Prevalência de lesões endodônticas em pacientes diabéticos e não diabéticos atendidos no Núcleo de Atenção Médica Integrada (NAMI). Fortaleza-CE, 2011.

\begin{tabular}{lccc}
\hline Lesões & Diabéticos (\%) & Não Diabéticos (\%) & $\boldsymbol{p}$ \\
LE & $36(90 \%)$ & $21(52,5 \%)$ & 0,0001 \\
DTE & $39(97,5 \%)$ & $39(97,5 \%)$ & $>0,05$ \\
DTE-LE & $31(77,5 \%)$ & $16(40 \%)$ & 0,001 \\
DSTE-LE & $28(70 \%)$ & $17(42,5 \%)$ & 0,03 \\
\hline
\end{tabular}

LE: dentes com lesão endodôntica; DTE: dentes tratados endodonticamente; DTE-LE: dentes tratados endodonticamente com lesão endodôntica; DSTE-LE: dentes sem tratamento endodôntico com lesões endodônticas.

Tabela III - Distribuição dos pacientes diabéticos e não diabéticos atendidos no Núcleo de Atenção Médica Integrada (NAMI) com total de dentes associado às lesões. Fortaleza-CE, 2011.

\begin{tabular}{lccc}
\hline \multirow{2}{*}{$\begin{array}{l}\text { Dotal de } \\
\text { Dentes }\end{array}$} & Diabéticos (\%) & Não Diabéticos (\%) & \multirow{2}{*}{$\boldsymbol{p}$} \\
\cline { 2 - 3 } & $\mathrm{n}=734$ & $\mathrm{n}=886$ & \\
\hline LE & $89(12,1 \%)$ & $37(4,1 \%)$ & 0,0189 \\
DTE & $115(15,6 \%)$ & $98(11,1 \%)$ & $>0,05$ \\
DTE-LE & $51(44,3 \%)$ & $17(17,3 \%)$ & 0,004 \\
DSTE-LE & $38(42,7 \%)$ & $20(20,4 \%)$ & 0,0045 \\
\hline
\end{tabular}

LE: dentes com lesão endodôntica; DTE: dentes tratados endodonticamente; DTE-LE: dentes tratados endodonticamente com lesão endodôntica; DSTE-LE: dentes sem tratamento endodôntico com lesões endodônticas. 
Nos dentes tratados endodonticamente, encontraramse, nos pacientes diabéticos, 44,3\% $(n=51)$ com lesões endodônticas; no grupo controle, apenas 17,3\% ( $\mathrm{n}=17)$ $(\mathrm{p}=0,0004)$.

Quanto aos dentes com lesões endodônticas e sem tratamentos endodônticos, encontraram-se 42,7\% (38) dos dentes em pacientes diabéticos; no grupo controle, o número foi bem inferior, apenas $20,4 \%(20)(p=0,0045)$ (Tabela III).

\section{DISCUSSÃO}

A Diabetes Mellitus é uma entidade patológica caracterizada por um distúrbio do metabolismo intermediário, especialmente no que tange aos carboidratos, levando ao aumento dos níveis séricos de glicose, a complicações metabólicas agudas potencialmente fatais e a uma série de complicações crônicas multissistêmicas, como insuficiência renal e doenças coronarianas ${ }^{(7,12)}$. Inclui ainda deficiências em combate a infecções, devido a deficiências leucocitárias ${ }^{(26)}$, e reparos e/ou cicatrizações de processos patológicos, devido à isquemia vascular ${ }^{(6)}$.

Na presente pesquisa, analisou-se, dentro de um banco de dados, a população de pacientes que apresentavam Diabetes Mellitus tipo II, geralmente diagnosticada em indivíduos com mais de 40 anos de idade, de ambos os sexos e obesos (80\%). O marco fisiopatológico dessa doença é a combinação de dois fatores: (i) resistência periférica à insulina e (ii) disfunção das células beta pancreáticas, que secretam quantidades de insulina insuficientes para o adequado controle metabólico ${ }^{(9,20)}$. Já está comprovado que é uma doença fruto da combinação de uma forte prédisposição genética com fatores ambientais e hábitos de $\operatorname{vida}^{(7)}$.

A avaliação do sucesso de tratamentos endodônticos é executada por vários parâmetros, entretanto, os pontos soberanos e mais consistentes são os clínicos, que estão diretamente ligados à manutenção de sintomatologia clínica e às evidências radiográficas que indicam o reparo de lesões endodônticas ${ }^{(5,20,27)}$. Pacientes diabéticos não compensados apresentam manutenção ou aumento de lesões endodônticas, mesmo quando não há associação com sintomatologia clínica dolorosa ${ }^{(10,15)}$, indicando falhas no processo de reparo apical ${ }^{(23)}$. Em estudo com população suíça, constatou-se que há maior incidência de lesões endodônticas em dentes tratados endodonticamente em pacientes diabéticos, independentemente da idade e do $\operatorname{sexo}^{(18)}$. Dessa forma, esses fatos associam a diabetes com a diminuição do sucesso do tratamento endodôntico, principalmente em casos em que a lesão apical é o fator de indicação do tratamento endodôntico ${ }^{(10,15,27)}$. Observouse no presente estudo uma maior prevalência de lesões periapicais visíveis radiograficamente nos pacientes diabéticos. Provavelmente, a ocorrência dessas lesões, em muitos casos, não decorre de insucesso endodôntico e local, e sim devido à deficiência da capacidade de reparo tecidual em função de alterações metabólicas ocasionadas pela diabetes, ou seja, uma deficiência sistêmica.

Devido ao fato de a Diabetes Mellitus afetar diretamente as respostas imunológicas e regenerativas, o processo de reparo da região apical também é afetado ${ }^{(15)}$. Consequentemente, pode causar uma superestimação dos índices de incidência e prevalência de lesões endodônticas em pacientes diabéticos que apresentam dentes tratados endodonticamente, quando comparados aos pacientes sem diabetes $^{(15)}$.

$\mathrm{Na}$ presente pesquisa, a maior parte dos pacientes era do sexo feminino, da mesma forma que em outros estudos $^{(10,15,23)}$. Sexo e idade são fatores que não influenciam em resultados associados à frequência de dentes tratados endodonticamente e presença de lesões endodônticas ${ }^{(19,25)}$.

Para a análise da presença de lesões endodônticas, o uso de radiografias periapicais ainda é a metodologia mais utilizada e difundida, permitindo uma análise criteriosa e com definição dessa região ${ }^{(2,5,18,23)}$. Provavelmente, em um futuro próximo, novos estudos passarão a utilizar a tomografia como padrão para evidenciar a presença de lesões endodônticas, pois permite detectar a lesão precocemente(28). Estudos excluíram a análise de regiões apicais de dentes com ausência ou falhas de restaurações coronárias, de dentes com canais tratados inadequadamente, e de dentes sobrepondo áreas anatômicas radiolúcidas ${ }^{(8,23)}$. Entretanto, acredita-se que esse fato influenciaria diretamente no perfil periapical dos pacientes do banco de dados investigado no atual estudo, dessa forma, todos os dentes presentes nos pacientes deste estudo foram analisados, com exceção dos terceiros molares.

A menor quantidade de dentes em pacientes diabéticos encontrada no presente estudo é compatível com outras investigações, as quais demonstram que a Diabetes Mellitus, principalmente quando não controlada, permite avanços severos da cárie dental e de doenças periodontais ${ }^{(5,8,14)}$. Pacientes que apresentavam abaixo de 7 dentes foram excluídos, pois estão dentro de uma classificação de doença periodontal severa ${ }^{(15)}$. Esse fato fez com que diminuísse a influência da doença periodontal no presente estudo.

Os resultados do atual estudo demonstraram maior ocorrência de lesões endodônticas em pacientes diabéticos. Esses resultados são concordantes com os resultados obtidos em trabalho epidemiológico da década de 1960, quando os autores sugerem que pacientes diabéticos apresentam falhas no processo de reparação tecidual apical, ou que esse processo ocorre de forma mais lenta do que em pacientes não diabéticos ${ }^{(24)}$. Posteriormente, demonstrou-se que realmente há a necessidade de maior tempo para reparo de lesões endodônticas em pacientes diabéticos ${ }^{(22)}$. 
Muitos modelos de estudo em animais têm sido desenvolvidos com o objetivo de correlacionar a Diabetes Mellitus com as características de lesões endodônticas. Em ratos, com diabetes induzida com estreptozotocina, foi demonstrada maior incidência de inflamação severa no ligamento periodontal, reabsorções radicular e óssea em relação ao grupo controles ${ }^{(29)}$. Reportaram que a reabsorção óssea alveolar foi mais severa, consequentemente, com maiores lesões endodônticas em ratos diabéticos tipo $\mathrm{II}^{(30)}$. Entretanto, os resultados do presente estudo, da mesma forma que os resultados obtidos anteriormente ${ }^{(15)}$, não apresentaram diferenças na escala PAI em dentes com lesões endodônticas em pacientes diabéticos e não diabéticos. Provavelmente, se esses estudos tivessem sido executados com uso de tomografia computadorizada, lesões em estágios iniciais e que não foram detectadas pelo uso de radiografias periapicais teriam sido detectadas.

Ao contrário dos resultados encontrados em outras pesquisas $^{(15,16)}$, na presente investigação, houve maior porcentagem de lesões endodônticas em dentes tratados endodonticamente nos pacientes diabéticos em relação ao grupo controle - o mesmo fato ocorreu em dentes com lesões endodônticas e sem tratamentos endodônticos. Pacientes diabéticos apresentaram aumento de doença periodontal em dentes tratados endodonticamente, demonstrando significativa redução no sucesso do tratamento endodôntico, porém, apenas nos casos em que havia presença de lesão apical inicialmente. Entretanto, o sucesso endodôntico geral da população analisada não foi influenciado pela presença da Diabetes Mellitus tipo II ${ }^{(10)}$.

Novos estudos epidemiológicos devem ser executados, buscando-se correlacionar dados dos tratamentos endodônticos em dentes submetidos a tratamentos periodontais, para que se possa constatar se há influências e inter-relação no índice de sucesso desses tratamentos em pacientes diabéticos.

\section{CONCLUSÃO}

Pode-se concluir que pacientes que apresentam Diabetes Mellitus tipo II apresentam maior prevalência de lesões endodônticas, independentemente das condições de tratamento do elemento dental. Esse fato pode afetar diretamente o acompanhamento e a determinação do sucesso endodôntico.

\section{REFERÊNCIAS}

1. Wolle CF, Zollmann LA, Bairros PO, Etges A, Leite CE, Morrone FB, et al. Outcome of periapical lesions in a rat model of type 2 diabetes: refractoriness to systemic antioxidant therapy. J Endod. 2013;39(5):643-7.
2. NG YL, Mann V, Gulabivala K. A prospective study of the factors affecting outcomes of non-surgical root canal treatment: part 2: tooth survival. Int Endod J. 2011;44(7):610-25.

3. Wang CH, Chueh LH, Chen SC, Geng YC, Hsiao CK, Chiang CP. Impact of diabetes mellitus, hypertension, and coronary artery disease on tooth extraction after nonsurgical endodontic treatment. J Endod. 2011;37(1):1-5.

4. Wang CH, Chueh LH, Chen SC, Feng YC, Hsiao CK, Chiang CP. Impact of diabetes mellitus, hypertension, and coronary artery disease on tooth extraction after nonsurgical endodontic treatment. J Endod. 2011;37(1):1-5.

5. Marotta PS, Fontes TV, Armada L, Lima KC, Rôças IN, Siqueira Junior JF. Type 2 Diabetes mellitus and the prevalence of apical periodontitis and endodontic treatment in an adult brazilian population. J Endod. 2012;38(3):297-300.

6. Vernillo AT. Dental considerations for the treatment of patients with diabetes mellitus. J Am Dent Assoc. 2003;134(Suppl 1):S24-33.

7. Blount CA, Leser C. Multisystem complications following endodontic therapy. J Oral Maxillofac Surg. 2012;70(3):527-30.

8. Lopez-Lopez J, Jané-Salas E, Estrugo-Devesa A, Velasco-Ortega E, Martin-González J, Segura-Egea J. Periapical and endodontic status of type 2 diabetic patients in Catalonia, Spain: a cross-sectional study. J Endod. 2011;37(5):598-601.

9. Moore PA, Zgibor JC, Dasanayake AP. Diabetes: a growing epidemic of all ages. J Am Dent Assoc. 2003;134(Suppl 1):S11-5.

10. Fouad AF, Burleson J. The effect of diabetes mellitus on endodontic treatment outcome: data from an electronic patient record. J Am Dent Assoc. 2003;134(1):43-51.

11. Lopes HP, Siqueira Jr JF. Endodontia: biologia e técnica. $3^{a}$ ed. Rio de Janeiro: Guanabara-Koogan; 2010.

12. Ship JA. Diabetes and oral health: an overview. J Am Dent Assoc. 2003;134(Suppl 1):S4-S10.

13. Lima SM, Grisi DC, Kogawa EM, Franco OL, Peixoto VC, Gonçalves Júnior JF, et al. Diabetes mellitus and inflammatory pulpal and periapical disease: a review. Int Endod J. 2013;46(8):700-9.

14. Taylor GW. The effects of periodontal treatment on diabetes. J Am Dent Assoc. 2003; 134(Suppl 1):S41-8. 
15. Arnold M, Riccuci D, Siqueira Júnior JF. Infection in a complex network of apical ramifications as the cause of persistent apical periodontitis: a case report. J Endod. 2013;39(9):1179-84.

16. Wang Z, McCauley LK. Osteoclasts and odontoclasts: signaling pathways to development and disease. Oral Dis. 2011;17(2):129-42.

17. Eriksen HM, Bjertness E, Brstavik D. Prevalence and quality of endodontic treatment in an urban adult population in Norway. Dent Traumatol. 1988;4(3):1226.

18. Imfeld TN. Prevalence and quality of endodontic treatment in an elderly urban population in Switzerland. J Endod. 1991;17(12):604-7.

19. Segura-Egea JJ, Castellanos-Cosano L, Machuca G, López-López J, Maartin-González J, Velasco-Ortega E, et al. Diabetes mellitus, periapical inflammation and endodontic treatment outcome. Med Oral Pathol Oral Cir Bucal. 2012;17(2):e356-61.

20. Cheung GSP, Wei WLL, McGrath C. Agreement between periapical radiographs and cone-beam computed tomography for assessment of periapical status of root filled molar teeth. Int Endod $\mathrm{J}$. 2013;46(10):889-95.

21. Maniglia-Ferreira C, Valverde GB, Silva Júnior JBA, Paula RCM, Feitosa JPA, Souza-Filho FJ. Clinical relevance of trans 1,4-polyisoprene aging degradation on the longevity of root canal treatment. Braz Dent J. 2007;18(2):97-101.

22. Falk H, Hugoson A, Thorstensson H. Number of teeth, prevalence of caries and periapical lesions in insulindependent diabetics. Scand J Dent Res. 1989;97(3):198206.

23. Britto LR, Katz J, Guelmann M, Heft M. Periradicular radiographic assessment in diabetic and control individuals. Oral Surg Oral Med Oral Pathol. 2003;96(4):449-52.
24. Bender IB, Seltzer S, Freedland J. The relationship of systemic diseases to endodontic failures and treatment procedures. Oral Surg Oral Med Oral Pathol. 1963;16(3):1102-15.

25. Ørstavik D, Kerekes K, Eriksen HM. The periapical index: a scoring system for radiographic assessment of apical periodontitis. Endod Dent Traumatol. 1986;2(1):20-34.

26. Cintra LT, Samuel RO, Azuma MM, Ribeiro CP, Narciso LG, de Lima VM, et al. Apical periodontitis and periodontal disease increase serum IL-17 levels in normoglycemic and diabetic rats. Clin Oral Investig. No prelo 2014. Disponível em: http://link.springer. com/article/10.1007\%2Fs00784-014-1192-7

27. Fouad AF. Diabetes mellitus as a modulating factor of endodontic infections. J Dent Educ. 2003;67(4):45967.

28. Ferreira MM, Carrilho E, Carrilho F. Diabetes mellitus and its influence on the success of endodontic treatment: a retrospective clinical study. Acta Med Port. 2014;27(1):15-22.

29. Kohsaka T, Kumazawa M, Yamasaki M, Nakamura H. Periapical lesions in rats with streptozotocin-induced diabetes. J Endod. 1996;22(8):418-21.

30. Iwama A, Nishigaki N, Nakamura K, Imaizumi I, Shibata N, Yamasaki M, et al. The effect of high sugar intake on the development of periradicular lesions in rats with type 2 diabetes. J Dent Res. 2003;82(4):3225.

\section{Endereço para correspondência: \\ Claudio Maniglia Ferreira \\ Curso de Odontologia da UNIFOR \\ Av. Washington Soares, 1321 \\ Bairro Edson Queiroz \\ CEP: 60.811-905 - Fortaleza - CE \\ E-mail: maniglia@unifor.br}

